\title{
Detection and genotype analysis of AmpC $\beta$-lactamase in Klebsiella pneumoniae from tertiary hospitals
}

\author{
XIANG-QUN LIU and YONG-RUI LIU
}

Department of Pneumology, Xuzhou City Hospital Affiliated to Xuzhou Medical College, Xuzhou, Jiangsu 221002, P.R. China

Received October 18, 2014; Accepted November 18, 2015

DOI: $10.3892 /$ etm.2016.3295

\begin{abstract}
The aim of the present study was to investigate the phenotype and genotype of plasmid-mediated AmpC (pAmpC) $\beta$-lactamase in Klebsiellapneumoniae and its antibiotic resistance. A total of 130 non-repetitive clinical isolates of Klebsiella pneumoniae, obtained from tertiary hospitals, were phenotypically screened for $\mathrm{pAmpC} \beta$-lactamase production with the cefoxitin disk diffusion test. $\beta$-lactamase genes in the screened isolates were detected using multiplex polymerase chain reaction (PCR); carbapenemase genes in $\mathrm{pAmpC} \beta$-lactamase-producing isolates that were resistant to imipenem were detected using PCR. Out of the 130 isolates of Klebsiella pneumoniae, 62 strains (47.7\%) were resistant to cefoxitin, including 14 strains (10.8\%) positive for pAmpC $\beta$-lactamase (DHA type), among which 12 strains $(85.7 \%)$ were susceptible to imipenem, and 2 strains, which were carrying Klebsiella pneumoniae carbapenemase (KPC)-2 gene, were resistant to imipenem. The $\mathrm{pAmpC} \beta$-lactamase-producing Klebsiella pneumoniae isolates from the tertiary hospitals were mainly of DHA-1 genotype, and the majority were susceptible to carbapenems; drug-resistant strains were associated with KPC-2 expression.
\end{abstract}

\section{Introduction}

AmpC $\beta$-lactamase, which is a type of cephalosporinase (1), is known to be responsible for antimicrobial resistance in gram-negative bacilli (2). The knowledge of potential risk factors for that resistance may help to limit its impact by enabling the implementation of effective control measures and judicious antimicrobial therapy (3). AmpC $\beta$-lactamases are either plasmid- or chromosomal-mediated (4). With regard to plasmid-mediated AmpC (pAmpC), no single phenotypic method is satisfactory for its detection; the combined application of phenotypic tests is necessary for the screening and confirmation of the presence

Correspondence to: Dr Xiang-Qun Liu, Department of Pneumology, Xuzhou City Hospital Affiliated to Xuzhou Medical College, 99 Huaihai Road, Xuzhou, Jiangsu 221002, P.R. China E-mail: lqyrcn@126.com

Key words: Klebsiella pneumoniae, plasmid, AmpC $\beta$-lactamase, carbapenemase of pAmpC-mediated resistance (5). Chromosomal-mediated AmpC hyperproducing Escherichia coli (E. coli) continues to be mostly susceptible to third-generation cephalosporins (6). Polymerase chain reaction (PCR) remains the gold standard for the detection of AmpC $\beta$-lactamases (7). As $\beta$-lactam antibiotics are widely used, the production of AmpC $\beta$-lactamases by bacteria results in considerable antibiotic resistance (8). Resistance to broad-spectrum $\beta$-lactams mediated by extended spectrum broad-spectrum $\beta$-lactamases (ESBLs) and AmpC $\beta$-lactamase enzymes is an increasing problem worldwide (9). In Portugal, 104/124 (83.9\%) of Enterobacteriaceae isolates that were resistant to third generation cephalosporins were also resistant to fourth generation cephalosporins (10). In comparison with ESBL-producing gram-negative bacilli, AmpC $\beta$-lactamase-producing strains show more extensive antibiotic resistance; $29 \%$ of Acinetobacter strains, for example, were found to produce both ESBL and AmpCenzymes (11). To be more specific, the majority of Acinetobacter baumannii isolates were producers of carbapenemase and metallo- $\beta$-lactamase (12). In a study of isolates collected between 2002 and 2008, DHA-1 was the most prevalent acquired AmpC (94\%), which was first identified in 2003 and was detected throughout the studied period in different institutions (13). Klebsiella pneumoniae is an important cause of nosocomial infection, and ESBLs and carbapenemases are a cause of emergency in multidrug-resistant (MDR) Klebsiella pneumoniae treatment (14). In a study of Enterobacter cloacae, a major nosocomial bacterium causing severe infections, the majority of the strains produced CTX-M type ESBLs, while a limited number produced SHV-type ESBLs, leading to the conclusion that the emergence of resistance genes is a public health problem (15). In a study conducted in a children's hospital, the proportion of Enterobacteriaceae isolates with broad-spectrum $\beta$-lactam resistance increased over a 3 -year period, mainly due to the emergence of a plasmid-mediated $\beta$-lactamase gene, bla $_{\mathrm{CMY}-2}(16)$. Therefore, the detection of pAmpC $\beta$-lactamases is important for the improvement of the clinical use of antibiotics. In the present study, the genotype of pAmpC $\beta$-lactamase from clinical samples of Klebsiella pneumoniae obtained from tertiary hospitals in Xuzhou was investigated, along with their antibiotic resistance.

\section{Materials and methods}

Strain source. A total of 130 non-repetitive clinical isolates of Klebsiella pneumoniae were obtained from patients in 
Table I. Primer sequences for the polymerase chain reaction amplification of AmpC $\beta$-lactamase target genes.

\begin{tabular}{|c|c|c|}
\hline Target gene & Primer sequence $\left(5^{\prime} \rightarrow 3^{\prime}\right)$ & Fragment length (bp) \\
\hline $\mathrm{bla}_{\mathrm{MOx}}$ & $\begin{array}{l}\text { F: GCTGCTCAAGGAGCACAGGAT } \\
\text { R: CACATTGACATAGGTGTGGTGC }\end{array}$ & 520 \\
\hline bla $_{\mathrm{CIT}}$ & $\begin{array}{l}\text { F: TGGCCAGAACTGACAGGCAAA } \\
\text { R: TTTCTCCTGAACGTGGCTGGC }\end{array}$ & 462 \\
\hline bla $_{\mathrm{DHA}}$ & $\begin{array}{l}\text { F: AACTTTCACAGGTGTGCTGGGT } \\
\text { R: CCGTACGCATACTGGCTTTGC }\end{array}$ & 405 \\
\hline $\mathrm{bla}_{\mathrm{ACC}}$ & $\begin{array}{l}\text { F: AACAGCCTCAGCAGCCGGTTA } \\
\text { R: TTCGCCGCAATCATCCCTAGC }\end{array}$ & 346 \\
\hline $\mathrm{bla}_{\mathrm{EBC}}$ & $\begin{array}{l}\text { F: TCGGTAAAGCCGATGTTGCGG } \\
\text { R: CTTCCACTGCGGCTGCCAGTT }\end{array}$ & 302 \\
\hline bla $_{\mathrm{FOX}}$ & $\begin{array}{l}\text { F: AACATGGGGTATCAGGGAGATG } \\
\text { R: CAAAGCGCGTAACCGGATTGG }\end{array}$ & 190 \\
\hline bla $_{\text {DHA }}$ (full-length primer) & $\begin{array}{l}\text { F: GGAATTCCACGGAAGGTTAATTCTGATG } \\
\text { R: GCAAGCTTTTATTCCAGTGCACTCA }\end{array}$ & 1140 \\
\hline
\end{tabular}

F, forward; R, reverse.

the Xuzhou First People's Hospital, the Affiliated Hospital of Xuzhou Medical College and Xuzhou Central Hospital (Xuzhou, China), between August and October 2012. The present study was conducted in accordance with the Declaration of Helsinki and was approved by the Ethics Committee of Xuzhou Medical College. Written informed consent was obtained from all participants.

Standard strains. E. coli strain ATCC 25922 (American Type Culture Collection, Manassas, VA, USA) and Enterobacter cloacae 029 M (National Center for Medical Culture Collections, Beijing, China) served as negative and positive controls, respectively, for the production of AmpC $\beta$-lactamase. E. coli strain ATCC 25922 and Klebsiella pneumoniae strain ATCC 13883 (National Center for Medical Culture Collections) served as negative and positive controls, respectively, for the production of carbapenemases.

Identification of bacteria. The clinical isolates were inoculated on blood agar medium (Oxoid, London, UK) and incubated at $35^{\circ} \mathrm{C}$ for $18-24 \mathrm{~h}$. A Vitek 32 automatic microbial analyzer (BioMerieux, Lyon, France) was then used for the identification and susceptibility testing of bacteria.

Screening for AmpC $\beta$-lactamase-producing strains. Strains were screened using a Kirby-Bauer disk diffusion test (17), in which cefoxitin (30 $\mu \mathrm{g}$; Oxoid) was used. According to the CLSI Antimicrobial Susceptibility Testing (AST) Standards (www.clsi.org/standards/micro/sub-ast/), isolates with an inhibitory zone diameter measuring $\leq 18 \mathrm{~mm}$ were suspected of being AmpC $\beta$-lactamase producers.

Multiplex PCR. AmpC $\beta$-lactamase genes were extracted using a Bacterial DNA Isolation kit (Tiangen Biochemical Technology Co., Ltd., Beijing, China). The screened clinical isolates were employed as templates for multiplex PCR (Shanghai Sangon Biological Engineering Technology and Services Co., Ltd., Shanghai, China) (18). Bi-directional DNA sequencing of the PCR products was performed by Shanghai Sangon Biological Engineering Co., Ltd. (Shanghai, China), using Basic Local Alignment Search Tool software (www.blast.ncbi.nlm.nih.gov/Blast.cgi) to determine the genotype. The sequencing results were identified using the GenBank database (www.ncbi.nlm.nih.gov/genbank/). The primer sequences are shown in Table I.

Detection of carbapenemase genes. In accordance with a previously reported method (19), PCR (CFX96 Touch Real-Time PCR system; Bio-Rad Laboratories, Inc., Hercules, CA, USA) was used to detect carbapenemase genes in pAmpC $\beta$-lactamase-producing isolates that were resistant to imipenem. The bacterial plasmid DNA extraction kit was provided by Tiangen Biochemical Technology Co., Ltd., as above. The primers were synthesized by Shanghai Sangon Biological Engineering Technology and Services Co., Ltd. (Shanghai, China), the sequences of which are reported in Table II. SYBR Green I (Thermo Fisher Scientific, Inc., Waltham, MA, USA) was used as fluorophore and ethidium bromide (Sigma-Aldrich, St. Louis, MO, USA) was used to visualize the DNA ladder (Sangon Biotech Co., Ltd., Shanghai, China). The results were captured and evaluated using a Gel Doc 2000 agarose gel imaging system (Bio-Rad Laboratories, Inc.).

\section{Results}

Preliminary screening. Out of the 130 Klebsiella pneumoniae isolates that were tested, 62 strains intermediate or resistant to cefoxitin were preliminarily screened as AmpC $\beta$-lactamase-producers, corresponding to a positive rate of $47.7 \%(62 / 130)$. 
Table II. Primer sequences for the polymerase chain reaction amplification of carbapenemase target genes.

\begin{tabular}{llc}
\hline Primer & \multicolumn{1}{c}{ Primer sequence $\left(5^{\prime} \rightarrow 3^{\prime}\right)$} & Fragment length $(\mathrm{bp})$ \\
\hline bla $_{\mathrm{KPC}}$ & F: GCTACACCTAGCTCCACCTTC & 920 \\
& R: ACAGTGGTTGGTAATCCATGC & 587 \\
bla $_{\mathrm{IMP}}$ & F: CTACCGCAGCAGAGTCTTTG & 261 \\
bla $_{\mathrm{VIM}}$ & R: AACCAGTTTTGCCTTACCAT \\
& F: AGTGGTGAGTATCCGACAG & \\
bla $_{\mathrm{SME}}$ & R: ATGAAAGTGCGTGGAGAC & 830 \\
& F: AACGGCTTCATTTTTGTTTAG & \\
\hline
\end{tabular}

F, forward; R, reverse.
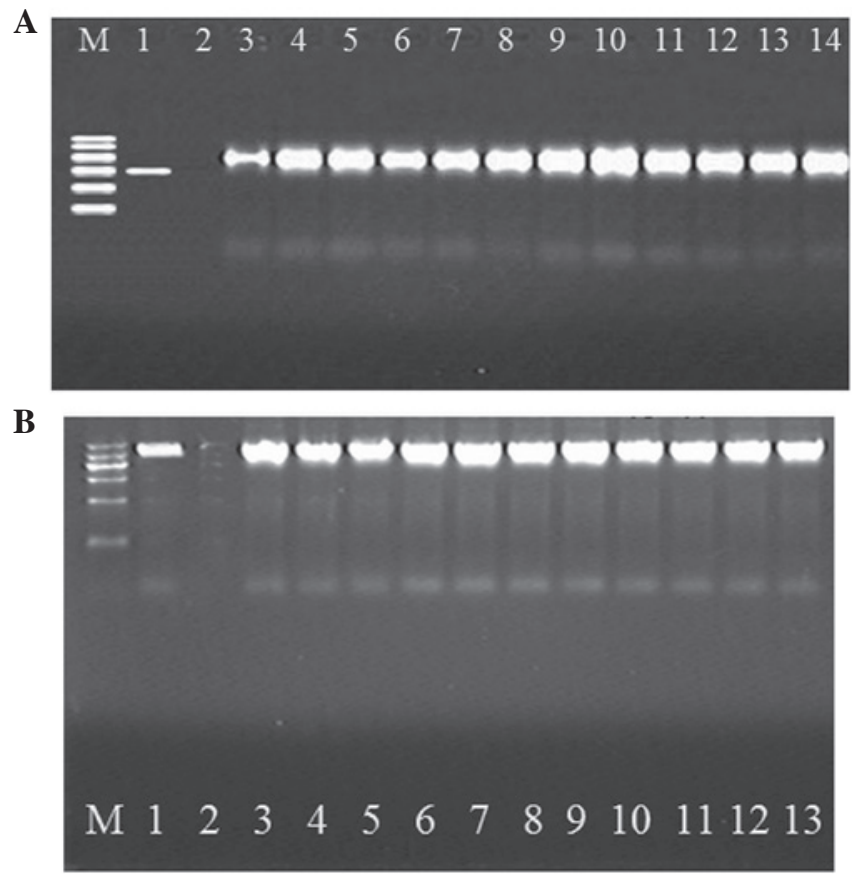

Figure 1. (A) Electrophoretogram of multiplex polymerase chain reaction (PCR) products of plasmid-mediated AmpC $\beta$-lactamase. M, DNA Marker (from top to bottom: 600, 500, 400, 300, 200 and $100 \mathrm{bp}$ ); 1, positive control; 2, negative control; 3-14, clinical isolates of Klebsiella pneumoniae carrying the bla $\mathrm{DHA}_{\mathrm{DHA}}$ gene. (B) Electrophoretogram of multiplex PCR products amplified with full-length primer. M, DNA marker (from top to bottom: 1,200, $900,700,500,300$ and $100 \mathrm{bp}) ; 1$, positive control; 2, negative control; $3-13$, clinical isolates of PCR products amplified with full-length primer.

Multiplex PCR. Multiplex PCR showed the amplification of a 405-bp fragment from 14 strains, which were thus identified as DHA type. The positive rate was $10.8 \%$ (14/130; Fig. 1A).

PCR amplification. The 14 bacterial strains that were identified by multiplex PCR screening were amplified with a full-length primer for the DHA type of AmpC $\beta$-lactamase, encoding a fragment of 1,140 bp (Fig. 1B).

Sequencing results. The PCR products obtained were purified, sequenced and identified to be of the DHA-1 type according to the GenBank database.
Susceptibility test. pAmpC $\beta$-lactamases (DHA-1)-producing Klebsiella pneumoniae were resistant to cefoxitin, ceftriaxone, ceftazidime, cefepime, ciprofloxacin, aztreonam, piperacillin-tazobactam, amikacin, levofloxacin and sulfamethoxazole, but susceptible to imipenem (Table III).

PCR amplification of carbapenemase. PCR revealed the amplification of an 2920 -bp fragment from two strains, which were thus identified as Klebsiella pneumoniae carbapenemase (KPC; Fig. 2). The PCR products obtained were purified, sequenced and verified to be of the KPC-2 type according to the GenBank database.

\section{Discussion}

Antibiotic resistance is a global concern (20), and the resistance to broad-spectrum $\beta$-lactams mediated by ESBLs and AmpC $\beta$-lactamase enzymes is an increasing problem worldwide (21). Klebsiella pneumoniae (22) is a common pathogen, from which pAmpC $\beta$-lactamase was first identified in South Korea in 1989. Since then, one or two new pAmpC $\beta$-lactamases have been reported annually (23), which are highly expressed (24) and can be transferred to other bacteria through conjugation and transformation, either by plasmid or chromosome, to cause antibiotic resistance (25). pAmpC $\beta$-lactamase-producing Klebsiella pneumoniae can cause serious infections (26), which makes anti-infective therapy more challenging. In Karnataka, a state in India, ESBL production was found to be $46.2 \%$ among clinical isolates of Klebsiella pneumoniae (27). In Islamabad, Pakistan, $54.55 \%$ of clinical isolates of Klebsiella pneumoniae, which were resistant to cefoxitin, were found to be pAmpC $\beta$-lactamase producers. Due to this high prevalence (28), further research into the distribution of pAmpC $\beta$-lactamases in Klebsiella pneumoniae and their drug susceptibility is required to improve the effectiveness of clinical antibiotic use.

In the present study, the cefoxitin disk diffusion test was used to preliminarily screen 130 Klebsiella pneumoniae isolates, out of which 62 strains were suspected of being AmpC $\beta$-lactamase-producers. Multiplex PCR was performed to detect the genotype of the screened strains and a 405-bp fragment was amplified from 14 strains, which were identified as being of DHA type according 
Table III. Susceptibility of 14 AmpC $\beta$-lactamase-producing Klebsiella pneumoniae strains.

\begin{tabular}{|c|c|c|c|c|c|c|}
\hline \multirow[b]{2}{*}{ Antibiotic } & \multicolumn{2}{|c|}{ Susceptible } & \multicolumn{2}{|c|}{ Intermediate } & \multicolumn{2}{|c|}{ Resistant } \\
\hline & Strains & Rate $(\%)$ & Strains & Rate $(\%)$ & Strains & Rate $(\%)$ \\
\hline FOX & 0 & 0 & 4 & 28.6 & 10 & 71.4 \\
\hline $\mathrm{CRO}$ & 3 & 21.4 & 0 & 0 & 11 & 78.6 \\
\hline CAZ & 2 & 14.3 & 1 & 7.1 & 11 & 78.6 \\
\hline FEP & 3 & 21.4 & 0 & 0 & 11 & 78.6 \\
\hline ATM & 3 & 21.4 & 0 & 0 & 11 & 78.6 \\
\hline TZP & 7 & 50 & 2 & 14.3 & 5 & 35.7 \\
\hline AMK & 4 & 28.6 & 0 & 0 & 10 & 71.4 \\
\hline CIP & 1 & 7.1 & 1 & 7.1 & 12 & 85.7 \\
\hline LVX & 3 & 21.4 & 0 & 0 & 11 & 78.6 \\
\hline IMP & 12 & 85.7 & 0 & 0 & 2 & 14.3 \\
\hline SMZ & 2 & 14.3 & 0 & 0 & 12 & 85.7 \\
\hline
\end{tabular}

FOX, cefoxitin; CRO, ceftriaxone; CAZ, ceftazidime; FEP, cefepime; ATM, aztreonam; TZP, piperacillin-tazobactam; AMK, amikacin; CIP, ciprofloxacin; LVX, levofloxacin; IMP, imipenem; SMZ, sulfamethoxazole.

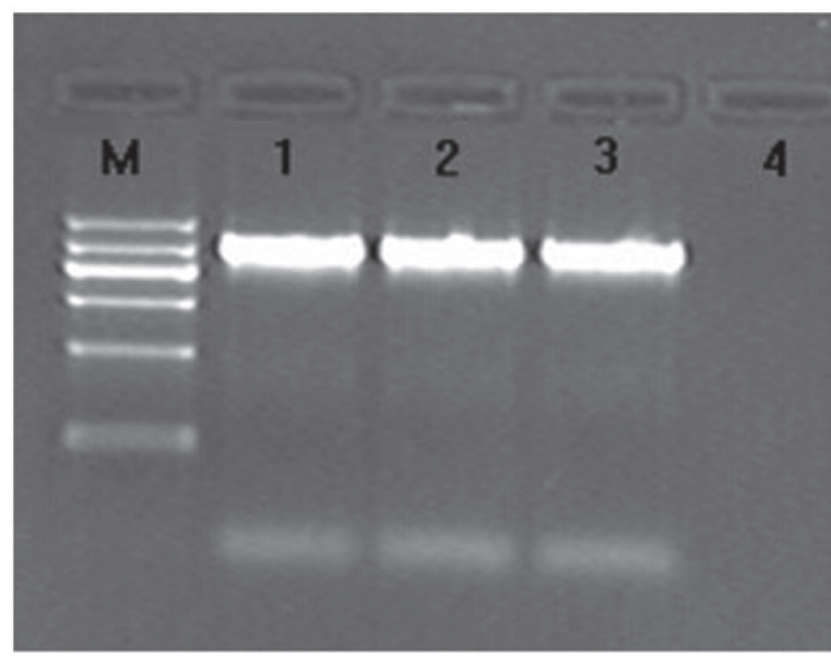

Figure 2. Electrophoretogram of PCR products of Klebsiella pneumoniae. carbapenemase. M, DNA Marker (from top to bottom: 1,200, 900, 700, 500, 300 and $100 \mathrm{bp}$ ); 1 and 2, two clinical isolates of bacteria; 3, positive control; 4 , negative control.

to the fragment size. These strains were amplified with full-length primer encoding a fragment of $1,140 \mathrm{bp}$, and the PCR products obtained were purified, sequenced and identified as being the DHA-1 type. The positive rate of pAmpC $\beta$-lactamase-producing Klebsiella pneumoniae in the samples from Xuzhou tertiary hospitals was $10.8 \%$, which was consistent with the report by Ding et al (29), but higher than the rate reported by Alvarez et al (30). Although CMY is the most common type of AmpC $\beta$-lactamase in the world, the DHA-1 and ACT types predominate in China (31). In the present study, the DHA-1 type was verified through genotype analysis of AmpC $\beta$-lactamase in Klebsiella pneumoniae, which was in accordance with a previous study (32); therefore, DHA-1 AmpC $\beta$-lactamase is speculated to be the main genotype in China.
The susceptibility test showed that AmpC-producing Klebsiella pneumoniae was resistant to multiple drugs, including third and fourth generation cephalosporins, such as ceftriaxone, ceftazidime and cefepime. The fourth generation cephalosporins can quickly pass through the outer membrane and are generally used to treat infections due to AmpC-producing bacteria (33). These bacteria, however, were found in the present study to be highly resistant to cefepime (78.6\%), and therefore the use of fourth generation cephalosporins is not recommended. AmpC-producing Klebsiella pneumoniae was also found to be highly resistant to quinolone and aminoglycoside antibiotics, including ciprofloxacin, levofloxacin and amikacin, but susceptible to carbapenem antibiotics since carbapenems are stable against $\beta$-lactamases and have high affinity for penicillin-binding proteins (34). Carbapenem antibiotics are therefore recommended for the treatment of infections caused by AmpC-producing strains of Klebsiella pneumoniae.

In the present study, there were two isolates of AmpC $\beta$-lactamase-producing Klebsiella pneumoniae that were found to be resistant to imipenem, which might indicate the production of KPC (34) or other antibiotic resistance mechanisms; therefore, PCR was applied to amplify carbapenemase genes, and confirmed that the strains were KPC-producing bacteria. KPC was first discovered in Klebsiella pneumoniae and named KPC-1 by Yigit et al (35), and is highly resistant to imipenem and meropenem. KPC has been reported worldwide, as well as in Zhejiang, Anhui, Tianjin and Shanghai in China (36). The present study has also identified KPC in Xuzhou, confirming that KPC is not limited by geographical boundaries. Klebsiella pneumoniae carrying two drug-resistance genes makes anti-infective therapy more difficult; therefore, it is recommended that tertiary hospitals in Xuzhou should strengthen the monitoring of AmpC $\beta$-lactamase in Klebsiella pneumoniae, and consider using carbapenems instead of fourth generation cephalosporins for the treatment of infections caused by AmpC-producing bacteria. 


\section{Acknowledgements}

The present study was supported by the 2012 Science and Technology Project in Xuzhou (grant no. XM12B019).

\section{References}

1. Neyestanaki DK, Mirsalehian A, Rezagholizadeh F, Jabalameli F, Taherikalani M and Emaneini M: Determination of extended spectrum beta-lactamases, metallo-beta-lactamases and AmpC-beta-lactamases among carbapenem resistant Pseudomonas aeruginosa isolated from burn patients. Burns 40: 1556-1561, 2014

2. Gupta G, Tak V and Mathur P: Detection of AmpC $\beta$ lactamases in gram-negative bacteria. J Lab Physicians 6: 1-6, 2014.

3. Belas A, Salazar AS, Gama LT, Couto N and Pomba C: Risk factors for faecal colonisation with Escherichia coli producing extended-spectrum and plasmid-mediated AmpC $\beta$-lactamases in dogs. Vet Rec 175: 202, 2014.

4. Wassef M, Behiry I, Younan M, El Guindy N, Mostafa S and Abada E: Genotypic identification of AmpC $\beta$-lactamases production in gram-negative bacilli isolates. Jundishapur J Microbiol 7: e8556, 2014.

5. Balıkçı H, Açıkgöz ZC, Güvenman S, Celikbilek N and Ozdem B Detection of plasmid-mediated AmpC beta-lactamase production in Escherichia coli and Klebsiella spp. isolates. Mikrobiyol Bul 48 . 82-93, 2014 (In Turkish).

6. Paltansing S, Kraakman M, van Boxtel R, Kors I, Wessels E, Goessens W, Tommassen $\mathrm{J}$ and Bernards A: Increased expression levels of chromosomal AmpC $\beta$-lactamase in clinical Escherichia coli isolates and their effect on susceptibility to extended-spectrum cephalosporins. Microb Drug Resist 21: 7-16, 2015.

7. Helmy MM and Wasfi R: Phenotypic and molecular characterization of plasmid mediated AmpC $\beta$-lactamases among Escherichia coli, Klebsiella spp., and Proteus mirabilis isolated from urinary tract infections in Egyptian hospitals. Biomed Res Int 2014: 171548, 2014.

8. Mohamudha PR, Harish BN and Parija SC: Molecular description of plasmid-mediated AmpC $\beta$-lactamases among nosocomial isolates of Escherichia coli \& Klebsiella pneumoniae from six different hospitals in India. Indian J Med Res 135: 114-119, 2012.

9. Grover N, Sahni AK and Bhattacharya S: Therapeutic challenges of ESBLS and AmpC beta-lactamase producers in a tertiary care center. Med J Armed Forces India 69: 4-10, 2013.

10. Jones-Dias D, Manageiro V, Ferreira E, Louro D; Antibiotic Resistance Surveillance Program in Portugal (ARSIP) participants Caniça M: Diversity of extended-spectrum and plasmid-mediated AmpC $\beta$-lactamases in Enterobacteriaceae isolates from Portuguese health care facilities. J Microbiol 52: 496-503, 2014.

11. Singla P, Sikka R, Deeep A, Gagneja D and Chaudhary U Co-production of ESBL and AmpC $\beta$-lactamases in clinical isolates of A. baumannii and $A$. lwoffii in a tertiary care hospital from northern India. J Clin Diagn Res 8: DC16-DC19, 2014.

12. Begum S, Hasan F, Hussain S and Ali Shah A: Prevalence of multi drug resistant Acinetobacter baumannii in the clinical samples from tertiary care hospital in Islamabad, Pakistan. Pak J Med Sci 29: 1253-1258, 2013.

13. Freitas F, Machado E, Ribeiro TG, Novais Â and Peixe L: Long-term dissemination of acquired AmpC $\beta$-lactamases among Klebsiella spp. and Escherichia coli in Portuguese clinical settings. Eur J Clin Microbiol Infect Dis 33: 551-558, 2014.

14. Tugal D, Lynch M, Hujer AM, Rudin S, Perez F and Bonomo RA: Multi-drug-resistant Klebsiella pneumoniae pancreatitis: A new challenge in a serious surgical infection. Surg Infect (Larchmt) 16: 188-193, 2015.

15. Souna D, Amir AS, Bekhoucha SN, Berrazeg M and Drissi M: Molecular typing and characterization of TEM, SHV, CTX-M, and CMY-2 $\beta$-lactamases in Enterobacter cloacae strains isolated in patients and their hospital environment in the west of Algeria. Med Mal Infect 44: 146-152, 2014.

16. Qin X, Zerr DM, Weissman SJ, Englund JA, Denno DM, Klein EJ, Tarr PI, Kwong J, Stapp JR, Tulloch LG and Galanakis E: Prevalence and mechanisms of broad-spectrum beta-lactam resistance in Enterobacteriaceae: A children's hospital experience. Antimicrob Agents Chemother 52: 3909-3914, 2008.
17. Andrews JM and BSAC Working Party On Susceptibility Testing ft: BSAC standardized disc susceptibility testing method. J Antimicrob Chemother 48: 43-57, 2001.

18. Pérez-Pérez FJ and Hanson ND: Detection of plasmid-mediated AmpC beta-lactamase genes in clinical isolates by using multiplex PCR. J Clin Microbiol 40: 2153-2162, 2002.

19. Nicola FG, Nievas J and Smayevsky J: Evaluation of phenotypic methods for the detection of KPC carbapenemases in Klebsiella pneumoniae. Rev Argent Microbiol 44: 290-302, 2012 (In Spanish).

20. Reardon S: Antibiotic resistance sweeping developing world. Nature 509: 141-142, 2014.

21. Voets GM, Platteel TN, Fluit AC, Scharringa J, Schapendonk CM, Stuart JC, Bonten MJ and Hall MA; National ESBL Surveillance Working Group: Population distribution of beta-lactamase conferring resistance to third-generation cephalosporins in human clinical Enterobacteriaceae in the Netherlands. PLoS One 7: e52102, 2012.

22. Bonnedahl J, Hernandez J, Stedt J, Waldenström J, Olsen B and Drobni M: Extended-spectrum $\beta$-lactamases in Escherichia coli and Klebsiella pneumoniae in Gulls, Alaska, USA. Emerg Infect Dis 20: 897-899, 2014

23. Markovska R, Schneider I, Marteva-Proevsk Y, Mitov I, Barernfeind A and Markova B: First detection of the AmpC beta-lactamase ACC-1 in a Klebsiella pneumoniae isolate in Bulgaria. J Chemother 24: 307-308, 2012.

24. Shanthi J and Balagurunathan R: Characterisation of heteroresistant subcolonies for MBL, AmpC genes in Klebsiella pneumoniae and Acinetobacter baumannii. Indian J Med Microbiol 32: 210-211, 2014.

25. Brenwald NP, Andrews J and Fraise AP: Activity of mecillinam against AmpC beta-lactamase-producing Escherichia coli. J Antimicrob Chemother 58: 223-224, 2006.

26. Pai H, Kang CI, Byeon JH, Lee KD, Park WB, Kim HB, Kim EC, Oh MD and Choe KW: Epidemiology and clinical features of bloodstream infections caused by AmpC-type-beta-l actamase-producing Klebsiella pneumoniae. Antimicrob Agents Chemother 48: 3720-3728, 2004.

27. Rao SP, Rama PS, Gurushanthappa V, Manipura R and Srinivasan K: Extended-spectrum beta-lactamases producing Escherichia coli and Klebsiella pneumoniae: A multi-centric study across Karnataka. J Lab Physicians 6: 7-13, 2014.

28. Shafiq M, Rahman H, Qasim M, Ayub N, Hussain S, Khan J and Naeem M: Prevalence of plasmid-mediated AmpC $\beta$-lactamases in Escherichia coli and Klebsiella pneumonia at tertiary care hospital of Islamabad, Pakistan. Eur J Microbiol Immunol (Bp) 3: 267-271, 2013 .

29. Ding H, Yang Y, Lu Q, Wang Y, Chen Y, Deng L, Wang A, Deng $Q$, Zhang $\mathrm{H}$, Wang $\mathrm{C}$, et al: The prevalence of plasmid-mediated AmpC beta-lactamases among clinical isolates of Escherichia coli and Klebsiella pneumoniae from five children's hospitals in China. Eur J Clin Microbiol Infect Dis 27: 915-921, 2008.

30. Alvarez M, Tran JH, Chow N and Jacoby GA: Epidemiology of conjugative plasmid-mediated AmpC beta-lactamases in the United States. Antimicrob Agents Chemother 48: 533-537, 2004.

31. Peng JH, Sun ZY, Zhou YL and Lv J: Study on the gene type of plasmid AmpC enzyme producing Klebsiella pneumonia. Zhong Hua Yi Yuan Gan Ran Xue Za Zhi 17: 1072-1075, 2007 (In Chinese)

32. Shi W, Li K, Ji Y, Jiang Q, Wang Y, Shi M and Mi Z: Carbapenem and cefoxitin resistance of Klebsiella pneumoniae strains associated with porin OmpK36 loss and DHA-1 $\beta$-lactamase production. Braz J Microbiol 44: 435-442, 2013.

33. Shi WF, Zhou J and Qin JP: Transconjugation and genotyping of the plasmid-mediated AmpC beta-lactamase and extended-spectrum beta-lactamase genes in Klebsiella pneumoniae. Chin Med J (Engl) 122: 1092-1096, 2009.

34. Queenan AM and Bush K: Carbapenemases: The versatile beta-lactamases. Clin Microbiol Rev 20: 440-458, 2007.

35. Yigit H, Queenan AM, Anderson GJ, et al: Novel carbapenem-hydrolyzing $\beta$-lactamase, KPC-1, from a carbapenem-resistant strain of Klebsiella pneumonia. Antimicrob Agents Ch 45: 1151-1161, 2001.

36. Zhao SP, Jiang MJ and Zong GZ: Comparison of detection methods for drug sensitivity of KPC-producing Klebsiella pneumonia. Zhong Hua Shi Yan He Lin Chuang Gan Ran Bing Za Zhi 6: 5-7, 2012. 